\title{
DESIGN OF A WIRE IMAGING SYNCHROTRON RADIATION DETECTOR:
}

\author{
J. Kent, J-J. Gomez-Cadenas, A. Bogan, M. King, W. Rowe, S. Watson, and C. Von Zanthier \\ University of California at Santa Cruz, Santa Cruz, CA 95064 \\ D. D. Briggs \\ Stanford Linear Accelerator Center, Stanford University, Stanford, CA 94309 \\ M. Levi \\ Lawrence Berkeley Laboratory, Berkeley, CA 94720
}

\section{A bstract}

This paper dacuments the design of a detector invented to measure the positions of synchrotron radiation beams for the precision energy spectrometers of the Stanford Linear Collider (SL,C).

The energy measurements involve the determination, on a pulse-by-pulse basis, of the separation of pairs of intense beams of syuchrotron pholons in the MeV energy range. The detector intercepts the beams with arrays of fine wires. The ejection of Compton recoil electrons results in charges being developed in the wires, thus enabling a determination of beam positions.

\section{INTRODUCTION}

The synchrotron radiation detectors documented here are part of the SLAC Linear Collider's precision energy spectrometers [1,2] Precise energy determinations are essential to the SLC physits program; e.g., for measurements of the mass of the $Z^{0}$. This introduction considers aspects of the energy spectromelers relevant to the design of our detectors.

Figure 1 is a schematic diagram of an SLC extractionline spectrometer. Two dipole magnets, one upstrean and one downstream of the opectrometer magnet, generate swathes of synchrotron radiation. The agle between these two synchrotron beams is inversely proportional to the SLC beam energy and proportional to the field strength of the spectrometer magnet. The angle is determined by measuring the separation between the pair of synchrotron beams at a known distance downstream from the magnetic center of the spectrometer magnet. The purpose of the Wire Imaging Synchrotron Radiation Detectors (WISRDs) is to monitor this separation-and hence the beam energy -on a pulse-to-pulse basis.

A WISRD is installed in both the electron and the positron energy spectrometers. Aside from mechanical details resulting from a $90^{\circ}$ rotation about the beam direttion, the positron spectrometer's WISRD is identical to

\footnotetext{
Work sopported by Departmeat of Energy contracts DEAC03-76SF00098, DE-AC03-76SF00515, and DE-AM03$76 \$$ F00010.
}

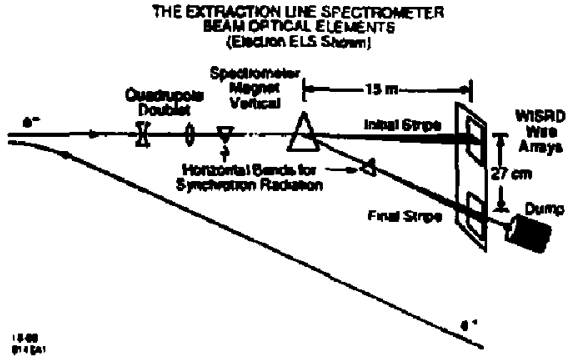

Fig. 1. Scbematic diagram of the (electron) extraction-line spectrometer beam optics and synchrotron beams.

the electron spectrometer's WISRD. For ciarity of presentation, only the electron spectrometer is explicitly considered here. Basically, the WISRD functions as follows. The speetrum of the two intense beams of synchrotion radiation are characterized by a critical energy of approximately $3 \mathrm{MeV}$. A fine wire intercepting one of these beams will develop a positive charge due to the ejection of Compton recoil electrons. The intensity of syachrotron photons is sufficient to directly induce a charge that can be measured electronically. The WISRD provides a determination of the separation between the two synchrotron beams by intercepting both beams with arrays of fine wires and measuring the charge induced on each wire; see Fig. 2.

Phntons in the MeV range are very penetrating. The spectrometer design provides two measures of the separation between the synchrotron beams. Just upst ream of the WISRD is a phosphorescent screen monilor (PSM) that aleo provides a high-precision measurement of the stripe separation. [3] The sligbt loes in synchrotron beam intensity due to the presence of an upetrean delector has practically no influence on the quality of data collected by the WISRD. This redundency, provided by monitoring the synchrotron beams with two independent detector systems. is a key feature of the design of the SLC spectrometers. 


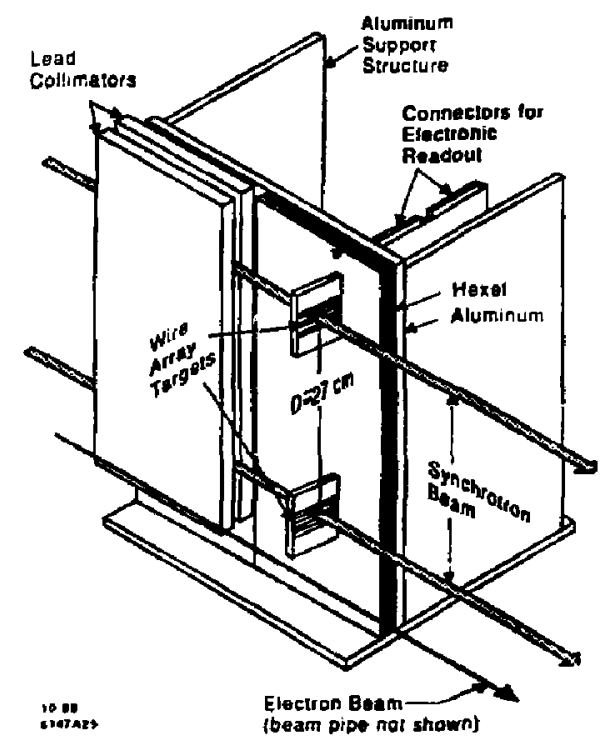

Fig. 2. A schematic view of a Wire Imaging Synchrotron Radiation Detector (WISRD), together with the SLC electron beam and the two beanus of synchrotron radiation.

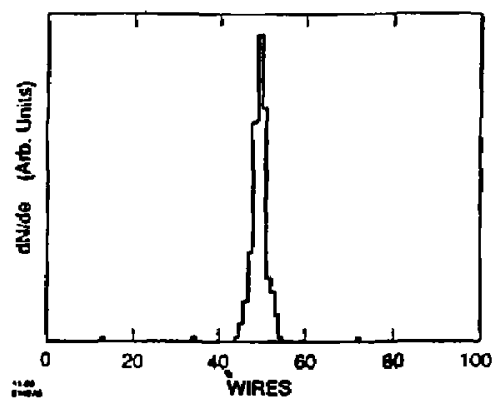

Fig. 3. Monte Carlo calculation of induced charge versus wire number. The above distribution corsesponds to an ideal primary beam of zero ernittanee, width, and dis persion. The tolal charge for a primary beam of $10^{10}$ electrons or positrons is calculated to be $180 \mathrm{fC}$ :

\section{The Physical Basis of the Detector}

The wite array targets are the heart of the detector shown in Fig. 2. The target arrays are composed of $75 \mu \mathrm{m}$ ciam copp - ( $5 \%$ beryllium) wirea with center-to-center spacing of $100 \mu \mathrm{m}$. There are 96 wires per arrsy. A sepatate channel of electronics is provided for each wire [1] This section considers the physical mechenim leading to af $^{-}$ nal generation on the wires and their implications for the design of the wire array targets as devicus for providing precision measurements of synchrot ron beam positions.

Figure 3 presents Monte Carlo predictions for the generated charge as a function of wire number for a wire array target exposed to a syachrotron beam Such a calculation involves three major phygical considerations which are sufficiently well known to allow quantilative predictions: properties of the beam of synchrotion photons, cross sections for the production of recoil electrons, and electron ranges in the wire material. The characteristic energy and intensity of the extraction-line spectroneters' synchrotron beams were unavailable in test heams.

Tbe properties of the incidept synchrotion photon beams have decisive influence on the design of the wire array targets. Many of the beam properties follow dirett]y from principles of electrodynamics. IS] Monte Carlo simulation of the synchrotron beams is straightforward for given assumptions regarding the intensity and beamı optic properties of the primary $e^{ \pm}$beam.

The critical energy of the photon spectrum is approximately $3 \mathrm{MeV}$, which follows from a primary beam energy of about $45 \mathrm{GeV}$ and a magnetic field strength of about 1.1 T. Synchrotron beams with such a high critical energy did not exist before the SLC. Because of this relatively high critical energy, Compton scattering dominates the generation of charges on the target wires.

Absorption of synchrotron photons due to the phowelectric effect is calculated to account for $20 \%$ of our signal. The photoelectric effect and Compton scattering contribute equally to the ejection of electrons from target wires by $250 \mathrm{keV}$ synchrotron photons. However, due to its atcep energy dependeace, the importance of the photoelectrie effeet decreases rapidly with incident photon energy. The importance of the photoelectric effect for the detector is further reduced by electron range effects.

The diameter of the target wires correspionds approximately to the range of a $300 \mathrm{keV}$ electron. Electron range is a rapidly increasing function of kinetic energy. The prob$a^{2}$-jlity that a recoil electron stops before exiting a wire is essentially unity for $100 \mathrm{keV}$ incident photons. Thus the physical mechanism of signal generation contains a "filter" that rejects photons with energies less than $\approx 200 \mathrm{keV}$. This is desirable because photons at the low end of the synchrotron spectrum are produced at greater emission angles with respect to the primary beam.

The recoil electrons produce $\delta$-rays within the target wires. Sorne of these $\delta$-rays will escape the wires. This effect is calculated to enbance the signal by $5 \%$.

The wire spacing of $100 \mu \mathrm{m}$ was chosen to permit the determination of a stripe centroid even in the presence of occasional dead channels. The syschrotron stripe profile shown in Fig. 3 is calculated for the case that the finite otripe width arises entirely from the finite production angles $(\approx 1 / \gamma)$ of synchrotron photons with respect to the momentum of the primary beam particles. For design tuning of the bean optics, one of the two gynchrotron beams is close to this limiting case. 
In general the stripe profile is a convolution of the profile in Fig. 3, with the spreads due to dippersion and apot size of the primary beam. The $100 \mu \mathrm{m}$-target wire spacing is optimal for our application; little would be gajned from a closer spacing.

The vertical geometrical acceptance-and hence the number of wires- was chosen to be elose to $1 \mathrm{~cm}$. This acceptance accommodates reasonable variations in the steering of the primary beam. The synchrotron swath profiles are always considerably narrower than this.

The high intensity of the synchrotron beams maines it unpecessary to have any gain mechanism within the detector. This leads to a robust detector with a linear signal respopse. Primary beam intensities are typically $10^{10}$ particles per pulse, which is calculated to induce a total charge of $180 \mathrm{fC}$ on a wire array target. Witb input capacitance from several meters of cable between the detector and the electronics, the ratio of signal-to-noise is of order $10^{2}$ with appropriate electronics.

Given the center-to-center wire spacing of $100 \mathrm{~mm}$, the $75 \mu \mathrm{m}$ wire diameter maximizes the volume of target material without rendering the techoical problem of maintaining electrical isolation between individual wires urmanageable, (A tilted wire-array-target geometry with a $100 \mu \mathrm{m}$ spacing, as seen by the bean, but a larger physical wire sparing-would have permitted larger target wire diamevers. For our application, this alcernative was judged to be an undesirable complication.) The choice of target wire material also affects the signal amplitude. The number of Compton recoil electrons produced in a wire is proportional to the density of electrons within the wire material, i.e., $\rho$ - Z/A. Copper was chosen as the target material because of its high density of electrons. The choice of copper over tungten was based in large part on the solderability and more ductile nature of copper wire.

The specifications for the wire array targets followed quickly from a quantitative understanding of the mechanism of signal production. The remainder of the WISRD design is mainly a matter of providing the wire array targets with the necessary mechanical support and alignmept, electrical connections, and electromegnetic shielding.

\section{The Wire Array Target Assembly}

The greatest technical challesge in the constsuction of the detectors was the mechanical degign and fabrication of the wire array targets. The gaps between neighboring vires are only $25 \mathrm{\mu m}$. A great deal of care was disected to the problem of avoiding accidental electrical contacto between the wires both in the target region and in the fanout for the electrical connections.

Figure 4 shows the wire target assembly built on a slab of machinable ceramic.|6] This materisl vias chosen because it is a radiation-hard rigid machinable insulator of moderate cost. The synchrotron beam pasees through the milled-out slot in the ceramic card before being intercepted by the wire array. Wires are glued in place at both ends of the target region by cersmic adhesive.[ग] Afler the

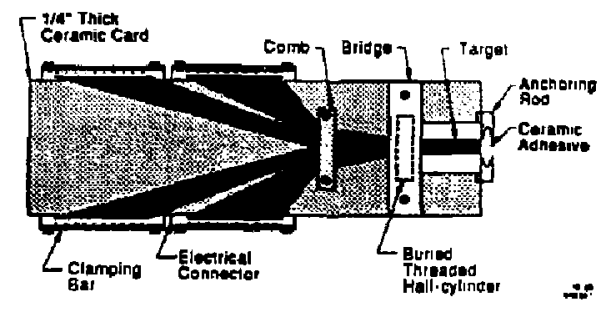

Fig. 4. Wire target array on its certamic card.

glue set, the target wires became mechanically part of the ceramic card.

The proximity of the primary electron beam and the lower synchrotron beam (see Fig. 2) restricts the space available to the anchor outer end of the wire array target. This motivaled gluing the target wires to a half-inch diameter MACOR []$^{[0]}$ rod mounted at the end of the slot milled in the ceramic card. The silhouette of the rod was reduced by $25 \%$ by milling away its inside edge. This design satisfied the space constraints while providing enough mechanical strength to support the tension of the wires.

Escential to the success of the project was the succeseful threading at a pitch of $100 \mu \mathrm{m}$ of half-inch MACOR rod. In addition to the anchoring rod previously discussed, the threaded MACOR rod was used to make a threaded half-cylinder that is imbedded in the bridge. It was these grooves in MACOR that aligned the target wires before gluing.

A custom winding jig was devjsed to string and hold the wires before the gluing step. Continuous lengths of wire were wrapped many times around the ceranic card. After the glue bad dried on both ends of the target array, the wire was cut at the anchoring rod. An acid etch was applied to remove electrical contacts between the closely packed wire ends. The comb shown in Fig. A provides a secondary pivot point for the mechanical fan-out of the wires; this also was essential to avoid accidental electrical contacts. For mechanical and electrical protection, the wires were potted in silicone rubber on the fan-out side of the bridge.

Contractions of the glue during drying and imperfections in the MACOR rod threads Jead to small deviations of the wires from their nominal positions. The final wire positions are known to better tban $10 \mu \mathrm{m}$. Data analyses may utilize a date buse of redundant measurements of the relative position of each wire within the target arrays.

\section{The WISRD SUPport Structure}

The structure seen in Fig. 2 satisfies the needs of the target arrays for support and alignment, electical connections, and electromagnetic shielding. Also the support atructure bolds the defining collimators for the sjichrotron beams. 
For our application, it is important that the distance between the pair of target arrays in Fig. 2 be known and stable to one part in ten-thousand (25 $\mu \mathrm{m})$. Stability is arhieved by making the two target arrays part of one rigid mechanical structure. The wires are glued to the ceramic cards which in turn are glued into an invar plate. The target separation is fixed even when subjected to reasonable temperature variations. Careful and redundant measurements in the shop before installation permit us to know the target separation within the desired accuracy. The invar plate is attached to a larger aluminum support structure to provide additional strength to the invar plate. $A$ layer of aluminum hexcel is laminated between the invar plate and the aluminum support structure with the various layers attached with metallic adhesive. This arrangement allows the aluminum structure to keep the invar flat. Sate that linear thermal expansions of the aluminum do not stress the invar plate.

The aluminum support structure serves a number of other purposes, including the following. The lead collimators. shown schematically in Fig. 2, define the $1 \mathrm{~cm}$ xidth of the synchrotron beams reaching the wire arry targets. Three large threaded rods (not shown) connect the aluminum support structure to the mounts in the machine tunnel. A number of tooling-ball holes in the eupport structure permit surveyors to measure the alignment of the WISRD. [9]

The WISRD is designed to be radiation hard. The detector operates in a high-radiation environment. The materials used to construct the WISRD, such as metals and ceramics, are radiation hard. The ceramic adhesive, used to glue the wires in place and to fasten the ceramic cards in the invar plate, is very fine alumina powder set with water; i.e., high-technology dried mud. This ceranic adhesive can be trusted to be radiation hard. The meakest link in radiation hardness is the plastic cable connectors shown in Fig. 4. To minimize out dependence on the mechanical strength of this plastic, the connectors are clamped in place with aluminum bars.

Electronic issues have a decisive influence on the mechanical design of the detectors. The SLC machine tunnel is an electromagnetically noisy environment. The high radiation levels at the WISRD force the associated frontend electronjes to be placed several meters from the detector. Th.js increases the potential for pickup and grounding problems and increases the equivalent noise charge.

To minimize electramagnetic pickup, the WISRD is provided with an RF shield which is distinet from signal ground. The signal conductors are completely surrounded by the signal ground which in turn is enclosed by the RF shield. Electrical contact is avoided between the RF shield and local grounds such as the beam pipe. This topology of conductors is continued in the cabling to the front-end electronies. Realizing this shielding and grounding philosophy was a major issue in the mechanical design of the WISRD.
The "onion" topology of signal, signal ground, and RF shield is realized as follows. All structures shown in Fig. 2 that are not part of the ceramic card assemblies (see Fig. 4) are part of a common signal ground. At the connectors on the ceramic cards, the target wires electrically continue as inner conductors of coaxial cables whose outer conductors are extentions of signal ground. The entire assembly shown in Fig. 2 is completely surrounded by an aluminum sheet metal box which is tile RF shield. It comprises two pieces that are electrically joined with the aid of metal mesh squeezed between iridited aluminum surfaces. This RF box is electrically connected to another RF box containing the electronics by shielding foil surrounding each of eight bundles of coaxial cables.

The signal conductors are completely surrounded by the signal ground to eliminate any direct capacitive coupling between the RF shield and the signal conductors. Not shown in Fig. 2 are metal shells that enclose the ceramic cards with signal ground. Sheet metal shells screwed onto the aluminum support structure surround the fan-out section. Metal cups screwed tightly, with an indium seal, onto the invar plate assure that the wires in the target area are completely surrounded by signal ground.

The metal cups protecting the wire arrays also permit control of the atmosphere surrounding the target. Both nilrogen and a rough vacuum have been used with success. A dry atmosphere is preferred to avoid corrosion of the wires and to avoid $a$ drop in the $A C$ resistivity due to humidity in the ceramic adhesive used to anchor the wires.

\section{Conctusions and Discussion}

Novel wire imaging synchrotion radiation detectors have been successfully designed and operated. The SLC energy apectromelers make use of two such devices. Preliminary data demonstrate that the detectors function as anticipated [10] The WISRDs have an important role to play in precision energy measurements for the SLC.

\section{ACKNOWLEDGMENTS}

Success depended on the considerable skill exercised by Frits Van Dyk and the UCSC machine shop. The au. thors wish to thank Gordon Bowden of SLAC for providing essential design ideas at the inception of the project. John Tinsman for his contributions to the development of the electronjes for the WISRDs, and Forest Rouse for his major contributions in the data acquisition sof urare during development and in sepvice.

\section{REFERENCES}

[1] J. Kent et al., Proc. of the IEEE Particle Accelerator Conf., Chicago, IL, March 20-23, 1989; SLAC-PUB4922.

[2] M. Levi et al, Nucl. Instrem. Methods A. SLAC PLB-4654; 
S. Watson at al, Proc. of the IEEE Particle Accelerator Conf.. Chicago. 1L. March 20-23. 1989; SLAC-PUB4908 .

[3] M. Levi et al., Proc. of the IEEE Particle Acceleralor Conf. Chicago, IL. March 20-23, 1989: SLAC-PL'B4921.

[4] D.D. Beiggs et at., Proc. of the Nuclear Scierre Symp. Orlanto, FL, November 9-11, 1988: SLAC-PLB-4737; LBL-26243.

[0] See, for example, J. D. Jackson. Clossical Electrodynamics.
[6] Cotronics 914. marthinable glass seramic.

[7] Cotronics 989. ceramic adhesive.

[8] ConNing Glassim.

(9] SLC Energy Spectrometer Sole Xios. 35 an. 56 . unpublished.

[10] MARK I1/SLC Note Nos. 242 and 243. unpublinhed.

\section{DISCLAIMER}

This repont was prepared as an accoupt of work sponsoted by an agency of the United States Government. Neither the Unitod States Government nor any agency thereor, nor any of their employeer, makes any warranily, express or implied, or assumes any lezal liability of responsibility for the aceuracy. completeness, or usefulness of any information, appasanes, proturt, or process disclosod, or represents that its use would not infringe privately awned rights. Refer. ence bereio to any speciftc commercial product, process, or service by irade name, trademart, mannfactorer, or otherwise does not necessarily constiture or imply its endorscment, reconmendation, of favoring by the Unitad States Government of any agency thereor. The views and opinions of authors expressed herein to not necessarily state of refleet those of the United States Goverameth of any agency thereof. 